\title{
Obtención y caracterización de recubrimientos de biovidrio sobre la aleación de Mg AZ31
}

\author{
Paula Toròs ${ }^{1}$, Franco Fontinovo Goyenechea ${ }^{1}$, \\ Ulises Gilabert $^{1,2}$, Marisa Sierra ${ }^{1}$, \\ María Cristina Di Stefano ${ }^{1}$
}

\author{
${ }^{1}$ Departamento de Ingeniería Química, Facultad Regional Buenos Aires, Grupo IDETQA, Universidad Tecnológica Na- \\ cional, Av. Medrano 951, Buenos Aires, Buenos Aires, Argentina. \\ e-mail: ptoros@frba.utn.edu.ar \\ ${ }^{2}$ SEGEMAR, Avda. General Paz 5445. Edificio 14, San Martín, Buenos Aires, Argentina \\ e-mail: ptoros@frba.utn.edu.ar
}

\section{RESUMEN}

El material ideal para una prótesis ósea metálica debe sufrir una corrosión gradual in vivo, sin liberación de componentes tóxicos, mientras actúa como soporte y facilita el crecimiento del tejido óseo, hasta que se complete su reemplazo por el hueso regenerado.

Las aleaciones de magnesio biodegradable han sido objeto de estudio debido a sus excelentes propiedades mecánicas y de biocompatibilidad. El obstáculo que estos materiales presentan es su elevada velocidad de corrosión. Una vez iniciado el proceso, se eleva el $\mathrm{pH}$ alrededor de los tejidos, interfiriendo con el crecimiento celular en la superficie de la aleación de magnesio.

La aplicación de recubrimientos protectores con el objetivo de mejorar la resistencia a la corrosión es una efectiva solución a este problema.

Una película protectora diseñada con este fin debe poseer las siguientes características: (i) suministrar la bioactividad requerida para facilitar la unión entre el hueso y el material (ii) ser lo suficientemente compacta para retardar la penetración del medio fisiológico a la aleación facilitando la regeneración del hueso (iii) poseer la suficiente fuerza de cohesión y adhesión entre el recubrimiento y el sustrato, evitando el agrietamiento de la película durante la implantación.

En este estudio se preparó por sol-gel el material denominado 45S5 Bioglass $\left(46.14 \% \mathrm{SiO}_{2}, 26.91 \% \mathrm{CaO}\right.$, $24.35 \% \mathrm{Na}_{2} \mathrm{O}, 2.60 \% \mathrm{P}_{2} \mathrm{O}_{5}, \%$ molar) y luego se depositó sobre piezas de aleación de magnesio AZ31 (3\% $\mathrm{Al}, 1 \% \mathrm{Zn}, 0.2 \% \mathrm{Mn}, \mathrm{Fe}<0.005 \%, \% \mathrm{~m} / \mathrm{m})$, previamente pulidas y lavadas en ultrasonido. Los recubrimientos se obtuvieron por la técnica de inmersión-emersión (“dip-coating”). Algunas de las muestras fueron sometidas a un pretratamiento con soluciones acuosas de $\mathrm{NaOH}$ caliente con el fin de suministrar una mayor adherencia del recubrimiento vítreo. Seguidamente fue realizado un tratamiento térmico en aire a dos temperaturas distintas, $400{ }^{\circ} \mathrm{C} \mathrm{y} 430^{\circ} \mathrm{C}$. Para evaluar las propiedades de los recubrimientos se determinaron las curvas de polarización correspondientes en medio fisiológico simulado (SBF).

Palabras clave: recubrimiento, anticorrosivo, magnesio, biovidrio.

\section{ABSTRACT}

The ideal material for a metal bone prosthesis must undergo a gradual corrosion in vivo, without releasing toxic components, while acting as a support and facilitating the growth of bone tissue, until a replacement is completed by the regenerated bone. .

Biodegradable magnesium alloys have been studied because of their excellent mechanical properties and biocompatibility. The obstacle that these materials have is its high corrosion rate. Once initiated the process, the $\mathrm{pH}$ around tissues rises, interfering with cell growth on the surface of the magnesium alloy.

In order to improve the corrosion resistance, the application of protective coatings as an effective solution to this problem is used.

A protective film designed for this purpose must have the following characteristics: (i) provide bioactivity required to facilitate bonding between the bone and the material (ii) be compact enough to retard penetration 
of physiological medium to the alloy facilitating regeneration bone (iii) to have sufficient cohesive strength and adhesion between the coating and the substrate to avoid the film cracking during implantation.

This study was prepared by sol-gel material called 45S5 Bioglass® $(46.14 \% \mathrm{SiO} 2,26.91 \% \mathrm{CaO}, 24.35 \%$ $\mathrm{Na} 2 \mathrm{O}, 2.60 \% \mathrm{P} 2 \mathrm{O}$, \% m) and was deposited on samples of AZ31 magnesium alloy (3\% $\mathrm{Al}, 1 \% \mathrm{Zn}, 0.2 \%$ $\mathrm{Mn}, \mathrm{Fe}<0.005 \%, \% \mathrm{~m} / \mathrm{m}$ ), previously polished and washed in ultrasound. The coatings were obtained by the technique of immersion-emersion (dip-coating). Some of the samples were in a pretreatment with aqueous solutions of hot $\mathrm{NaOH}$, in order to provide greater adherence of the vitreous coating. Then a thermal treatment was conducted in air at different temperatures, $400^{\circ} \mathrm{C}$ and $430^{\circ} \mathrm{C}$. To determine the coatings' corrosion resistance, the polarization curves in simulated physiological medium (SBF) were determined.

Keywords: coating, anticorrosive, magnesium, bioglass.

\section{INTRODUCCIÓN}

Las aplicaciones del magnesio y de sus aleaciones[1-3] ha crecido en los últimos años, por ejemplo, en las industrias automotriz, aeroespacial, de comunicaciones como así también de accesorios deportivos, debido a la elevada relación peso-resistencia mecánica, baja densidad y estabilidad dimensional. Dado que además de sus excelentes propiedades mecánicas son biocompatibles, han sido objeto de estudio para su uso en prótesis óseas [4-7]. El obstáculo que estos materiales presentan es su elevada velocidad de corrosión. Una vez iniciado el proceso, se eleva el $\mathrm{pH}$ alrededor de los tejidos, interfiriendo con el crecimiento celular en la superficie de la aleación de magnesio. Una efectiva solución a este problema es la aplicación de recubrimientos protectores. Una película protectora diseñada con este fin debe poseer las siguientes características: (i) suministrar la bioactividad requerida para facilitar la unión entre el hueso y el material, (ii) ser lo suficientemente compacta para retardar la penetración del medio fisiológico a la aleación facilitando la regeneración del hueso (iii) poseer la suficiente fuerza de cohesión y adhesión entre el recubrimiento y el sustrato, evitando el agrietamiento de la película durante la implantación . Las películas del material denominado 45S5 Bioglass $®$ (46.14\% $\mathrm{SiO}_{2}, 26.91 \% \mathrm{CaO}, 24.35 \% \mathrm{Na}_{2} \mathrm{O}, 2.60 \% \mathrm{P}_{2} \mathrm{O}_{5}, \%$ ) cumplen con estas condiciones [8-12]. En este trabajo se ha aplicado, mediante la técnica de inmersión-emersión ("dip-coating"), biovidrio obtenido por sol-gel, sobre aleaciones de Mg AZ31. Entre las ventajas del método sol-gel podemos mencionar: (1) menor temperatura de fabricación, que tiene un papel significativo en el costo y la calidad del producto, (2) un control más eficiente de composición y homogeneidad, y (3) mayor área superficial, que implica un incremento en la velocidad de disolución en el fluido corporal y aumenta la bioactividad del material resultante.

El objetivo de este trabajo es determinar las mejores condiciones de aplicación, mediante la técnica mencionada, de biovidrio obtenido por sol-gel sobre Mg AZ31[13]. Se han publicado recientemente trabajos tendientes a lograr una mejor adhesión del recubrimiento sobre la aleación, a partir de modificar la superficie del sustrato. Estos tratamientos previos son básicamente de tres tipos: inmersión en un medio alcalino, inmersión en un medio ácido y conversión superficial. En este trabajo hemos analizado el efecto del tratamiento alcalino. Cuando una aleación de Mg se somete a una solución de $\mathrm{pH}$ mayor a 11, se recubre de una capa estable de $\operatorname{Mg}(\mathrm{OH})_{2}$, que mejora la adherencia del recubrimiento de biovidrio, por sus propiedades hidrofílicas. La humectabilidad de la superficie plana y limpia de magnesio no es buena, pudiendo generar grietas y mala adherencia de la película, debido a las diferencias entre los coeficientes de dilatación térmica entre ambos. Esto se evita mediante la formación de la capa de $\mathrm{Mg}(\mathrm{OH})_{2}$. Con el fin de determinar el efecto del pretratamiento, éste se aplicó sólo sobre algunas muestras. Finalmente se analizó el comportamiento de las muestras con pretratamiento y libres de él. Sobre todas las muestras se efectuó un tratamiento térmico posterior, a dos temperaturas distintas $\left(400^{\circ} \mathrm{C}\right.$ y $\left.430^{\circ} \mathrm{C}\right)$, a fin de evaluar el efecto sobre la calidad del recubrimiento obtenido. Se observó que durante el tratamiento térmico que la estructura vítrea se consolida, aumenta la densidad, debida a la contracción de volumen y se logra una reducción de la tensión residual, generada en la interfaz entre el revestimiento y el sustrato.

\section{MATERIALES Y MÉTODOS}

Se trabajó con muestras de aleación de magnesio AZ31 (3\% Al, 1\% Zn, 0.2\% Mn, Fe<0.005\%, \%m/m). El material fue pulido con papel abrasivo de SiC de distinta granulometría: $120 \mu \mathrm{m}, 320 \mu \mathrm{m}, 600 \mu \mathrm{m}, 1000 \mu \mathrm{m}$ y $1200 \mu \mathrm{m}$. A continuación las muestras fueron lavadas en ultrasonido. Se efectuaron tres baños sucesivos, de 10 minutos cada uno, en acetona, alcohol etílico y agua destilada. Sobre algunas muestras se efectuó un pretratamiento con solución de $\mathrm{NaOH} 1 \mathrm{M}$ y una temperatura de $80^{\circ} \mathrm{C}$, durante una hora, con el fin de suministrar una mayor adherencia del recubrimiento vítreo . 
La solución precursora del biovidrio 45S5 se preparó a partir de una solución de HNO3 1M a la cual se añadieron sucesivamente, a temperatura ambiente y con agitación constante $\mathrm{NaNO}_{3}, \mathrm{Ca}\left(\mathrm{NO}_{3}\right)_{2}$, tetraetil orto silicato (TEOS) y fosfato de trietilo (TEP). La agitación se mantuvo durante 3 horas.

En la aplicación de los recubrimientos mediante dip-coating se emplearon dos velocidades inmersiónemersión: $4 \mathrm{~cm} / \mathrm{min}$ y $22 \mathrm{~cm} / \mathrm{min}$. En cada caso se efectuaron un baño, 2 baños y 3 baños, de 80 segundos cada uno, con una pausa de un minuto entre baños.

Las muestras se mantuvieron 24 horas expuestas al aire, a temperatura ambiente. Seguidamente fue realizado un tratamiento térmico en aire. A fin de evaluar el efecto de la temperatura, a las muestras tratadas con $\mathrm{NaOH}$ se las sometió a dos temperaturas distintas durante el tratamiento térmico de $400{ }^{\circ} \mathrm{C} \mathrm{y} 430^{\circ} \mathrm{C}$

Se efectuó el ensayo DTA-Tg , análisis termogravimétrico ,el cual permite determinar, por tratamiento térmico controlado en una termobalanza, las variaciones de peso que experimenta un sólido con la temperatura. Se llevó a cabo en las muestras libre de recubrimiento y recubierta con biovidrio a fin de establecer eventuales sucesos térmicos en los procesos de cocción de los recubrimientos y determinar la temperatura límite superior para consolidar los recubrimientos. También se realizaron ensayos de DSC-Tg (Termogravimetría simultánea-calorimetría diferencial de barrido) sobre el biovidrio. En ambos casos, se utilizó un equipo de análisis térmico simultáneos TA-Q600 en atmósfera de aire sintético.

Se efectuó el ensayo de cinta adhesiva. Para el mismo se utilizó una cinta de adhesividad comprendida entre 6 y 7,5 N/cm. Se presionó la cinta adherida al sustrato con un rodillo de goma de aproximadamente $2 \mathrm{~kg}$ de peso, mediante dos pasadas sucesivas. Sujetando firmemente el extremo no pegado, se tiró bruscamente de la cinta con un ángulo comprendido entre $45^{\circ}$ y $90^{\circ}$. [14]

Los recubrimientos se caracterizaron mediante la determinación de las curvas de polarización potenciodinámicas. Se empleó un potenciostato/galvanostato Teq_4, controlado por microprocesador que opera en el rango de corriente que va desde $5 \mathrm{nA}$ FS hasta $250 \mathrm{~mA}$ FS. Los ensayos potenciodinámicos se efectuaron a una velocidad de barrido de $50 \mathrm{mV} / \mathrm{s}$, desde $-2000 \mathrm{mV}$ a $0 \mathrm{mV}$. Se empleó una celda convencional de vidrio Pyrex, de tres electrodos. Se utilizó un electrodo de referencia de calomel saturado y un contraelectrodo de platino. Las curvas de polarización se determinaron en medio fisiológico simulado (SBF) $(0.85 \% \mathrm{NaCl}$ $\left., 0.04 \% \mathrm{KCl}, 0.034 \% \mathrm{CaCl}_{2} .2 \mathrm{H}_{2} \mathrm{O}, \% \mathrm{~m} / \mathrm{m}\right)$ a $37^{\circ} \mathrm{C}$.

\section{RESULTADOS Y DISCUSIÓN}

En la figura 1 se presenta el ensayo DTA y Tg de la muestra libre de recubrimiento (línea continúa) y recubierta con biovidrio (línea de trazos). Puede observarse que hasta alrededor de $530{ }^{\circ} \mathrm{C}$ no se produce ningún cambio térmico detectable ni en el sustrato desnudo ni en el material recubierto. Alrededor de esa temperatura comienza la oxidación masiva del material metálico en la atmósfera oxidante en la que se realizó el ensayo lo que se pone de manifiesto por un aumento de la masa y un intenso cambio exotérmico. Este resultado determina la temperatura límite superior para consolidar térmicamente el recubrimiento en atmósfera oxidante. En cuanto al ensayo de la cinta adhesiva, el recubrimiento permaneció adherido al sustrato.

Cabe destacar que en la figura 1 se muestran las curvas de la muestra recubierta hasta $630{ }^{\circ} \mathrm{C}$ debido a que no se obtiene ninguna información adicional a mayores temperaturas. Alrededor de $575^{\circ} \mathrm{C}$ comienza a aumentar rápidamente la masa debido a la oxidación de la aleación metálica. Esa transformación térmica, por su intensidad, impide la detección de cualquier otro evento térmico que pueda ocurrir a temperaturas mayores que $630^{\circ} \mathrm{C}$. 


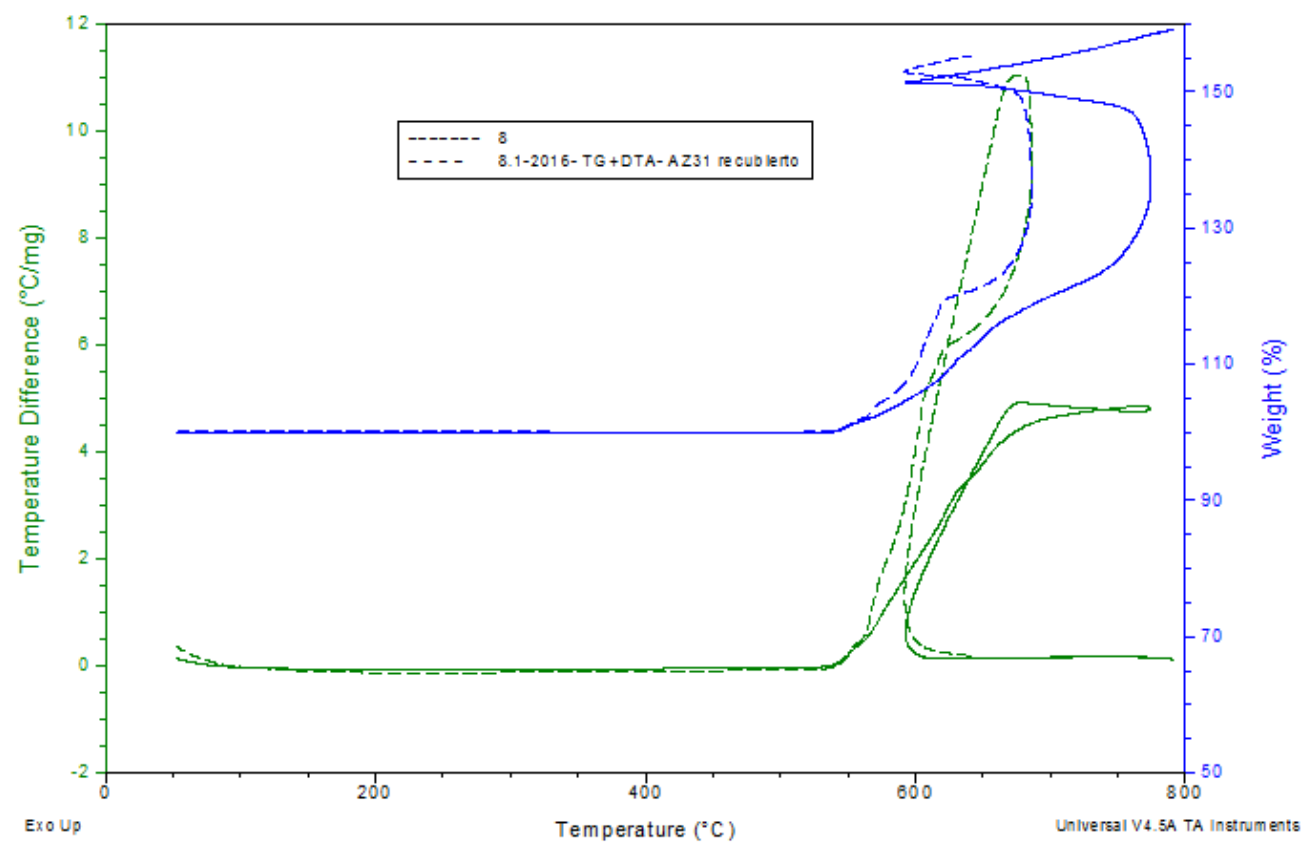

Figura 1: DTA y Tg de la aleación AZ31 libre de recubrimiento y cubierta con biovidrio. En función de la temperatura y la variación de masa.

En la figura 2 se pueden ver los resultados del ensayo de DSC y Tg del biovidrio sólo en atmósfera oxidante. Las transformaciones térmicas más destacables son las siguientes: el pico endotérmico de 115,21 ${ }^{\circ} \mathrm{C}$ se debe a la remoción de alcohol y agua, a $397,10^{\circ} \mathrm{C}$ hay otro pico endotérmico suave debido a la transición vítrea del material. Otro pico débil exotérmico probablemente se deba a la generación de cierta cantidad de una fase cristalina. Finalmente el pico exotérmico a $625,10^{\circ} \mathrm{C}$ se debe a la descomposición de los nitratos presentes en las materias primas. Los biovidrios obtenidos por sol-gel tienen una temperatura de transición vítrea menor si se la compara con la de los vidrios obtenidos por fusión junto con una mayor mesoporosidad y área superficial. Esto implica la posibilidad de eliminar más fácilmente la porosidad del recubrimiento.

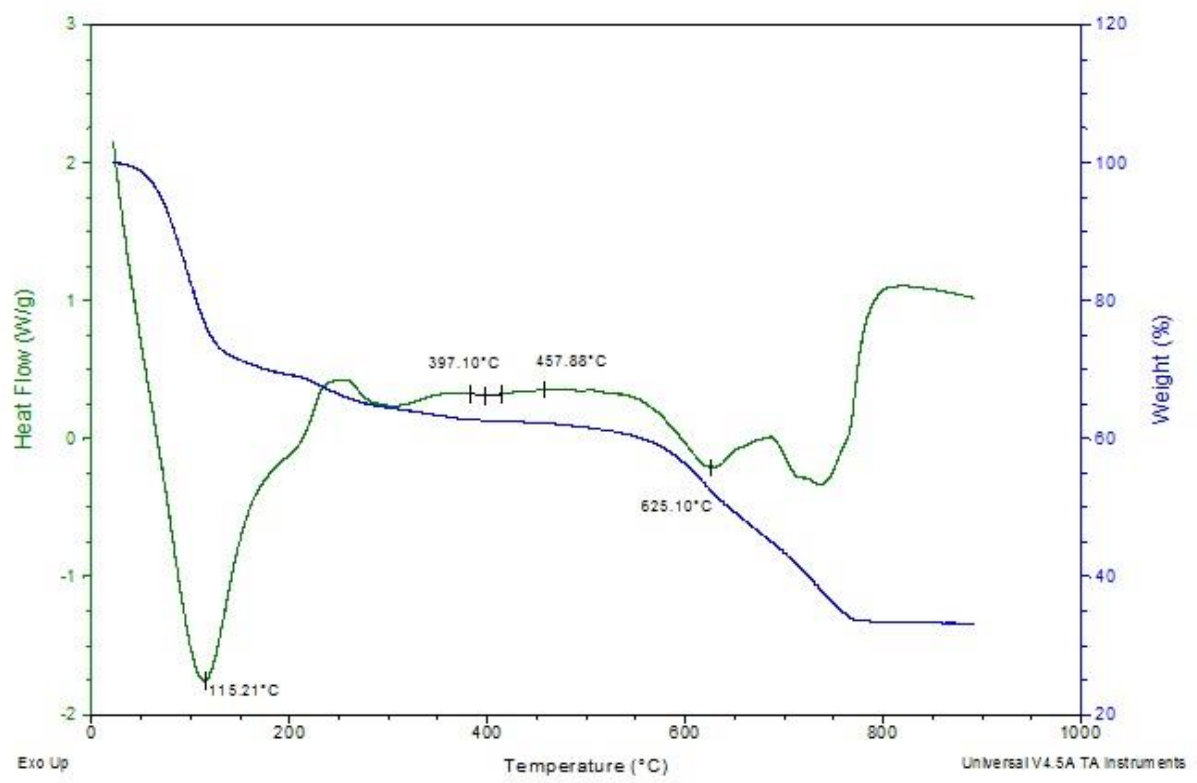

Figura 2: DSC y Tg de la aleación AZ31 libre de recubrimiento y cubierta con biovidrio. 
En la figura 3 se presenta el efecto del pretratamiento en solución de $\mathrm{NaOH}$ en los resultados de los ensayos electroquímicos para muestras tratadas térmicamente a $400^{\circ} \mathrm{C}$. En la figura 4 , en cambio, se presentan los ensayos electroquímicos de las muestras pretratadas con $\mathrm{NaOH}$ a $400{ }^{\circ} \mathrm{C}$ y $430{ }^{\circ} \mathrm{C}$. [9]

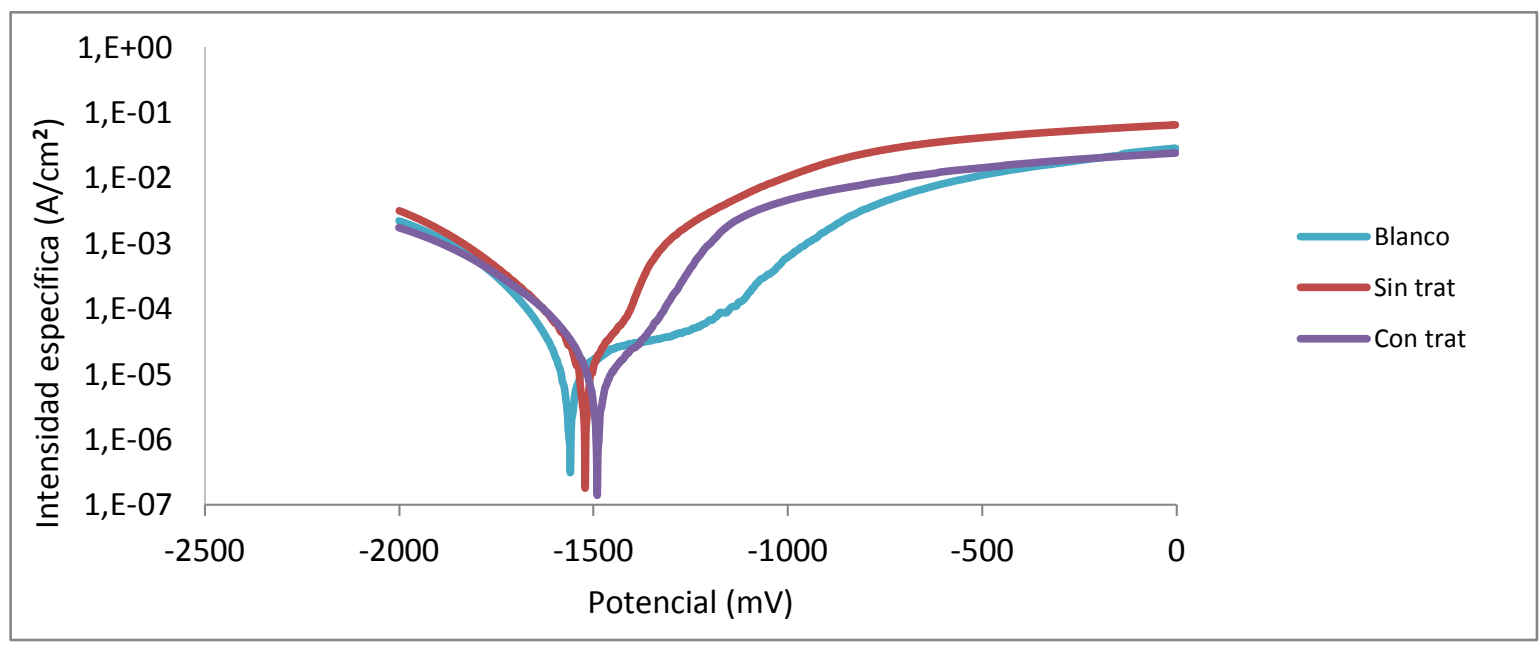

Figura 3: Efecto del pretratamiento con $\mathrm{NaOH}$, tratamiento térmico a $400{ }^{\circ} \mathrm{C}$

En la figura 3 puede verse que la muestra tratada con solución de $\mathrm{NaOH}$ evidencia un desplazamiento hacia valores mayores del potencial de corrosión y una disminución de valor de $\mathrm{i}^{0}$, aunque este último efecto no resulta tan significativo.

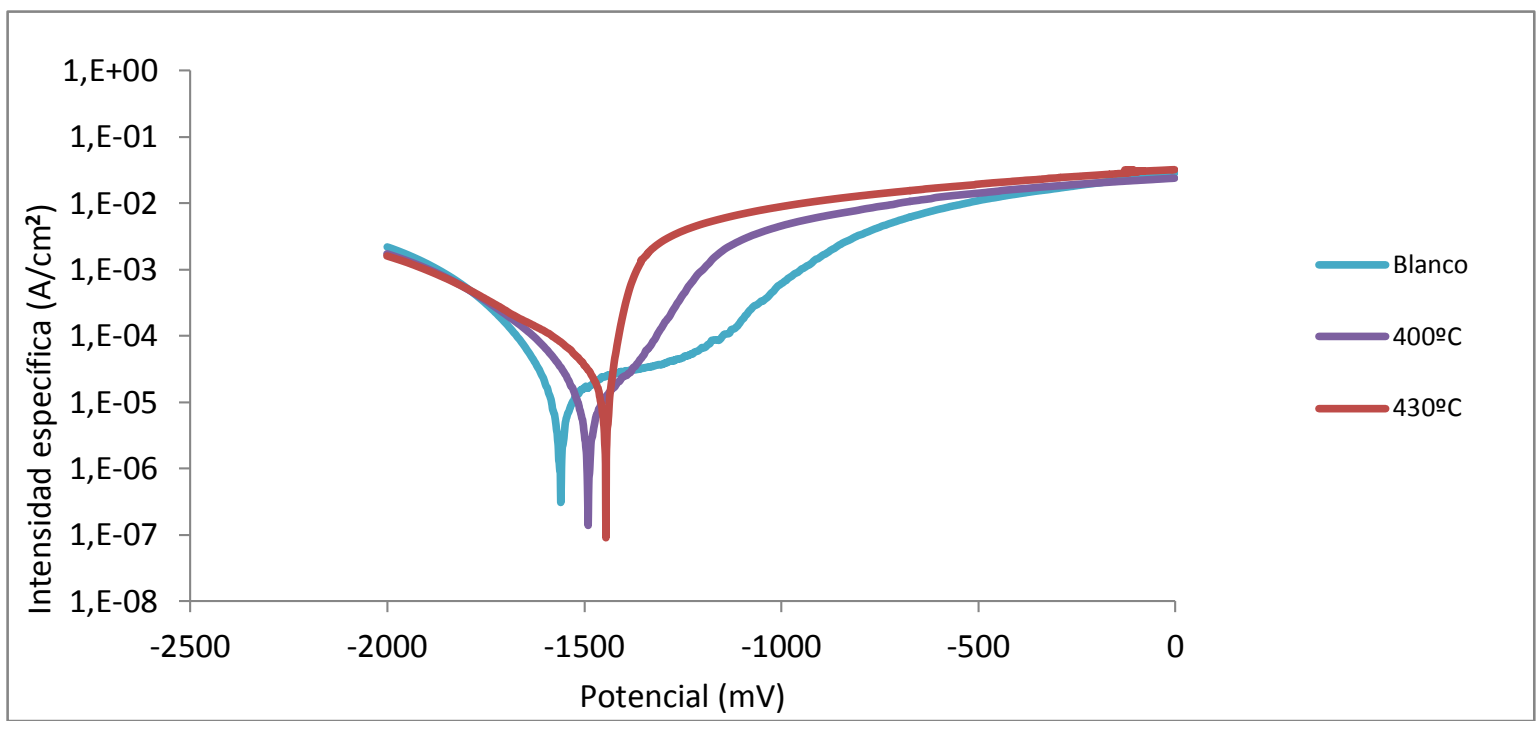

Figura 4: Efecto de la temperatura sobre las muestras pretratadas con $\mathrm{NaOH}$.

En la figura 4 se representan las curvas correspondientes a los ensayos potenciodinámicos de aleación AZ31 sin tratar (blanco), y dos muestras pretratadas con $\mathrm{NaOH}$ y recubiertas con biovidrio, cuya diferencia estriba en la temperatura del tratamiento térmico a $400{ }^{\circ} \mathrm{C}$ (trazo morado) y tratamiento térmico a $430{ }^{\circ} \mathrm{C}$ (trazo rojo). Se observa un desplazamiento hacia valores mayores del potencial de corrosión y una disminución de valor de $\mathrm{i}^{0}$, más significativo en el caso de la muestra tratada a mayor temperatura.

\section{CONCLUSIONES}

En los ensayos efectuados se observan los siguientes efectos:

- La inmersión de la aleación en solución $1 \mathrm{M}$ de $\mathrm{NaOH}$, con el efecto de la formación de una capa de $\mathrm{Mg}(\mathrm{OH})_{2}$ adherida a la superficie del sustrato, mejora la adherencia del recubrimiento de biovidrio. 
- El aumento en la temperatura del tratamiento térmico mejora la resistencia a la corrosión del recubrimiento de biovidrio.

De estas observaciones se puede concluir que el recubrimiento de biovidrio logra una mayor adherencia al ser aplicado sobre una superficie en la que se ha generado una precipitación de $\mathrm{Mg}(\mathrm{OH})_{2}$ y que una mayor temperatura en el tratamiento térmico consolida su estructura, evidenciado en los parámetros determinados en los ensayos potenciodinámicos, que expresan una mayor resistencia a la corrosión.

\section{BIBLIOGRAFÍA}

[1] LIU, L.J. "Corrosion of magnesium and its alloys", Corrosion Science, v.51,PP. 1733-1737, 2009.

[2] SHAW, BA. "Corrosion resistance of magnesium alloys", In: Stephen D, editor. ASM handbook volume 13a: corrosion: fundamentals, testing and protection. UK: ASM International, 2003.

[3] ESMAILY, M. "Fundamentals and advances in magnesium alloy corrosion" Progress in Materials Science v.89, pp. 92-193, 2017

[4] WITTE, F. "In vivo corrosion of four magnesium alloys and the associated bone response", Biomaterials v.26, n.17, pp.3557-63, 2005.

[5] WITTE, F. "In vitro and in vivo corrosion measurements of magnesium alloys", Biomaterials, v.27, n.7, pp.1013-8, 2006.

[6] WITTE, F. "Degradable biomaterials based on magnesium corrosion", Materials Science, v.12, pp. 6372,2008 .

[7] XU, L. P. "In vivo corrosion behavior of $\mathrm{Mg}-\mathrm{Mn}-\mathrm{Zn}$ alloy for bone implant: application", J Biomed Mater Res A, v83A, pp.703-11, 2007.

[8] ZHAO, H., "The influence of alkali pretreatments of AZ31 magnesium alloys on bonding of bioglassceramic coatings and corrosion resistance for biomedical applications", Ceramics International, v. 41, pp. 4590-4600, 2015.

[9] KOKUBO, T., "How useful is SBF in predicting in vivo bone bioactivity", Biomaterials, v. 27, pp. 29072915, 2006.

[10] PIRAYESH, H. \& NYCHKA, J. A., "Sol-gel synthesis of bioactive glass-ceramics 45S5 and its in vitro dissolution and mineralization behavior", Journal of American Ceramic Society, v. 96, pp. 1643-1650, 2013

[11] HUANG, K. "Preparation and characterization of mesoporous 45S5 bioactive glass-ceramic coatings on magnesium alloy for corrosion protection”, Journal of Alloys and Compounds ,v.580, pp.290-297, 2013.

[12] REN, M. G., "Influence of heat treatment on crystallization and corrosion behavior of calcium phosphate glass coated AZ31 magnesium alloy by sol-gel method", Journal of Non-Crystaline Solids, v. 369, pp. 69-75, 2013.

[13] GRAY, J. E., "Protective coatings on magnesium and its alloys - a critical review", Journal of Alloys and compounds, v. 336, pp. 88-113, 2002.

[14] ASTM D-3359, Standard Test Methods for Measuring Adhesion by Tape Test. 